\title{
Persistent Organic Pollutants and Heavy Metals and the Importance of Fish as a Bio-Indicator of Environmental Pollution
}

\author{
Tamer Tashla ${ }^{1}$, Radivoj Prodanović ${ }^{1}$, Jelana Bošković ${ }^{1}$, Milena Žuža ${ }^{2}$, Dragan Soleša ${ }^{1}$, Dragana Ljubojević ${ }^{3}$ and \\ Nikola Puvača ${ }^{1 *}$ \\ ${ }^{1}$ Department of Engineering Management in Biotechnology, University Business Academy, Faculty of Economics and Engineering \\ Management, Serbia
}

${ }^{2} J o h n$ Naisbitt University, Faculty of Bio farming, Serbia

${ }^{3}$ Scientific Veterinary Institute "Novi Sad", Serbia

Received: 眥 October 16, 2018; Published: 制 October 22, 2018

*Corresponding author: Nikola Puvača, University Business Academy, Faculty of Economics and Engineering Management, Department of Engineering Management in Biotechnology, Novi Sad, Serbia

\section{Opinion}

Nowadays water pollution is the burning issue all over the world. Aquatic ecosystems are frequently contaminated with different toxicants through anthropogenic activities, and some of them such as metals may be naturally present and essential in low concentration but toxic and harmful in higher concentrations. Having in mind that not all chemical forms of pollutants are equally bioavailable, and some pollutants can be accumulated in living organisms to a greater extent than others, there is a need to study the levels of pollutants in the organisms to be able to predict the environmental risk. Thus, chemical analyses of the tissues of aquatic organisms are used as a routine approach in studies of aquatic pollution, providing a temporal integration of the levels of pollutants with biological relevance at higher concentrations than those present in water or sediment, and facilitating their quantification [1]. Fish are among the group of aquatic organisms which represent the largest and most diverse group of vertebrates. A number of characteristics make them excellent experimental models for toxicological research, especially for the contaminants which are likely to exert their impact on aquatic systems [2]. Due to feeding and living in the aquatic environments fish are particularly vulnerable and heavily exposed to pollution because they cannot escape from the detrimental effects of pollutants. Fish, in comparison with invertebrates, are more sensitive to many toxicants and are a convenient test subject for indication of ecosystem health. Heavy metals are produced from a variety of natural and anthropogenic sources. In aquatic environments, heavy metal pollution results from direct atmospheric deposition, geologic weathering or through the discharge of agricultural, municipal, residential or industrial waste products. Heavy metals are able to disturb the integrity of the physiological and biochemical mechanisms in fish that are not only an important ecosystem component, but also used as a food source. Previous studies have shown that marine and farmed fish and shellfish are significant contributors to consumer intake of some contaminants due to their presence in the aquatic environment and their accumulation in the flesh of fish and shellfish. The objective of this article is to describe the effects of different persistent organic pollutants and heavy metals on the fish used as bioindicator of environmental pollution. Fish have been found to be good indicators of water contamination in aquatic systems because they occupy different trophic levels; they are of different sizes and ages and in comparison with invertebrates, are also more sensitive to many toxicants [3]. Last but not least, fish are the final chain of aquatic food web and an important food source for human. Therefore, some toxicants in aquatic environments can be transferred through food chain into humans.

\section{Persistent Organic Pollutants and Their Effect on Fish Target Organs}

\section{Gills}

The fish gills are multifunctional organs involved in ion transport, gas exchange, acid-base regulation and waste excretion. Given that the gills accounts for well over $50 \%$ of the surface area of a fish it is not surprising that one of the major target organs for waterborne toxicants is the gill. The gills are regarded as the important site for direct uptake from the water, whereas the body surface is generally assumed to play a minor role in xenobiotics uptake of fish. Thus, in teleost fish the gills are most frequently utilized in bioaccumulation studies and the pathological damage 
produced allows the toxicity of the environment to be defined, making fish highly suitable for evaluating the health of aquatic systems [4]. Fish metabolism, acting principally through the gills can be seriously damaged since toxicant incorporation occurs mainly through this respiratory organ. Furthermore, the fish gills are very sensitive to physical and chemical alterations of the aquatic medium such as: temperature, acidification of the water supply due to acid rain, salts and heavy metals, and to any change in the composition of the environment which is an important indicator of waterborne toxicants. fish gills are the main route of penetration of toxicants into the fish organism, thus they are the first organs which come in contact with environmental pollutants and are also sensitive subjects for identifying the effects of water toxicants on fish organisms. The fish gills can accumulate bioavailable pollutants, and their measurement on gills can reflect the speciation of pollutants, and in particular metals in water, therefore, they are a useful tool for assessing bioavailability of elements in water [5].

\section{Liver and Kidney}

Once the toxicants cross the biological barriers and enter the bloodstream, they will reach and accumulate in the internal organs of fish. Numerous studies have quantified contaminants in fish organs to evaluate environmental quality, seeking causal relationships with fish health, and, based on these, the liver is likely to be the best choice, followed by the kidney and gills. The liver is reported to be the primary organ for bioaccumulation and thus, has been extensively studied in regards to the toxic effects of xenobiotics. The liver is also a target organ due to its large blood supply which causes noticeable toxicant exposure. In addition, liver is a detoxification organ and it is essential for both, the metabolism and the excretion of toxic substances in the body. The vertebrate kidney is the main organ involved in the maintenance of body fluid homeostasis [6]. The morphology and function of the kidney have been modified through evolution to fulfill different physiological requirement and the widest range of kidney types is found in fishes. The kidney, together with the gills and intestine, are responsible for excretion and the maintenance of the homeostasis of the body fluids and, besides producing urine, act as an excretory route for the metabolites of a variety of xenobiotics to which the fish may be exposed. many studies showed that different toxicants accumulate mainly in metabolic organs such as the liver and kidney which can lead to many histological alterations. Levels of heavy metals such as lead, copper, cadmium, and zinc in marine fish have been extensively documented. These metals tend to distribute differentially between the liver and kidney and other organs, most likely because of metalbinding proteins such as metallothionein's in the metabolic organs [7].

\section{Fish Meat}

The fish meat is a very important, valuable and recommended food in the human nutrition due to low content of fat and high content of proteins and mineral substances as well as optimal ratio of unsaturated fatty acids with cardioprotective effect [8]. On the other hand, fish muscle may be the depositary for different contaminants, which occur in the water ecosystem. Such environmental pollutants are dioxins and PCBs, heavy metals, and organochlorine pesticides are a global threat to food safety, thus fish meat could lose these properties due to environmental contamination. Hydrobionts can bioaccumulate many of these contaminants potentially making seafood of concern for chronic exposure to humans. The metal concentrations in the water are positively correlated with the concentrations in fish tissues, but some research has founded that the metal concentrations in the sediments are the most important factor for their levels in the aquatic biota [9]. Consumption of fish contaminated with heavy metals have deleterious effects on human health which was widely acknowledged after a series of events in the period from 1953 to 1960 when several thousand people died in Japan as a result of poisoning caused by the consumption of mercury contaminated fish. Therefore, concern regarding the presence of heavy metals and other contaminants in seafood has arisen during the last decades.

\section{The Moust Important Heavy Metals for Fish as a Water Pollutants}

The contamination of heavy metals and metalloids in water and sediment, when occurring in higher concentrations, is a serious threat because of their toxicity, long persistence, and bioaccumulation and bio magnification in the food chain. Fishes are considered to be most significant bio monitors in aquatic systems for the estimation of metal pollution level, they offer several specific advantages in describing the natural characteristics of aquatic systems and in assessing changes to habitats. In addition, fish are located at the end of the aquatic food chain and may accumulate metals and pass them to human beings through food causing chronic or acute diseases. Studies from the field and laboratory works showed that accumulation of heavy metals in a tissue is mainly dependent on water concentrations of metals and exposure period; although some other environmental factors such as water temperature, oxygen concentration, $\mathrm{pH}$, hardness, salinity, alkalinity and dissolved organic carbon may affect and play significant roles in metal's accumulation and toxicity to fish [10]. Heavy metals are known to induce oxidative stress and carcinogenesis by mediating free reactive oxygen species. In general, metals can be categorized as biologically essential and non-essential. The nonessential metals such as $\mathrm{Al}, \mathrm{Cd}, \mathrm{Hg}$, Sn and $\mathrm{Pb}$ have no proven biological function, and their toxicity rises with increasing concentrations. Essential metals such as $\mathrm{Cu}, \mathrm{Zn}, \mathrm{Cr}, \mathrm{Ni}, \mathrm{Co}$, Mo and Fe on the other hand, have a known important bilogical roles in toxicity. The deficiency of an essential metal can therefore cause an adverse health effect, whereas its high concentration can also result in negative impacts which are equivalent to or worse than those caused by non-essential metals [11]. The toxicity of metals to fish is significantly affected by the 
form in which they occur in water. The ionic forms of metals or simple inorganic compounds are more toxic than complex inorganic or organic compounds. The toxic action of metals is particularly pronounced in the early stages of fish development and adversely affects various metabolic processes in developing fish, resulting in developmental retardation, morphological and functional deformities, or death of the most sensitive individuals [10]. Heavy metals produce toxic effects at high concentrations, and thus could be considered as risk factors for several diseases [11]. Heavy metals are able to disturb the integrity of the physiological and biochemical mechanisms in fish that are not only an important ecosystem component, but also used as a food source [8]. Previous studies have shown that marine and farmed fish and shellfish are significant contributors to consumer intake of some contaminants due to their presence in the aquatic environment and their accumulation in the flesh of fish and shellfish.

Biomarkers can offer additional biologically and ecologically relevant information - a valuable tool for the establishment of guidelines for effective environmental management. So, it can be stated that fish biomarkers are necessary for monitoring environmentally induced alterations to assess the impact of xenobiotic compounds such as heavy metals on fish. Also, it is recommended that treatment of all kinds of wastewaters, sewage and agricultural wastes must be conducted before discharge into the aquatic systems. Also, enforcement of all articles of laws and legislations regarding the protection of aquatic environments must be taken into considerations.

\section{References}

1. Yancheva V, Velcheva I, Stoyanova S, Georgieva E (2015) Fish in Ecotoxicological Studies. Eco Balk 7(1): 149-169.
2. Souza IC, Duarte ID, Pimentel NQ Rocha LD, Morozesk M, et al. (2013) Matching metal pollution with bioavailability, bioaccumulation and biomarkers response in fish (Centropomus parallelus) resident in neotropical estuaries. Environ Pollut 180: 136-144.

3. Mendil D, Demirci Z, Tuzen M, Soylak M (2010) Seasonal investigation of trace element contents in commercially valuable fish species from the Black sea, Turkey. Food Chem Toxicol 48(3): 865-870.

4. Playle RC, Dixon DG, Burnison K (2011) Copper and cadmium binding to fish gills: modification by dissolved organic carbon and synthetic ligands. Can J Fish Aquat Sci 50: 2667-2677.

5. Georgieva E, Stoyanova S, Velcheva I,Yancheva V (2014) Histopathological alterations in common carp (Cyprinus carpio L.) gills caused by thiamethoxam. Braz Arch Biol Technol 57(6): 991-996.

6. Monteiro DA, Rantin FT, Kalinin AL (2013) Dietary intake of inorganic mercury: bioaccumulation and oxidative stress parameters in the neotropical fish Hoplias malabaricus. Ecotoxicol 22(3): 446-456.

7. Siscar R, Koenig S, Torreblanca A, Sole M (2014) The role of metallothionein and selenium in metal detoxification in the liver of deep-sea fish from the NW Mediterranean Sea. Sci Total Environ 467: 898-905.

8. Ljubojević D, Ćirković M, Novakov N, Puvača N, Aleksić N, Lujić J, Jovanović R (2014) Comparison of meat quality of tench, Tinca tinca, reared in extensive and semi-intensive culture systems. J Appl Ichthyol 30: $50-57$

9. Widianarko B, Van Gestel CA, Verweij RA, Van Straalen NM (2000) Associations between trace metals in sediment, water, and guppy, Poecilia reticulata (Peters), from urban stream of Semarang, Indonesia. Ecotoxicol Environ Saf 46(1): 101-107.

10. Jitar O, Teodosiu C, Oros A, Plavan G, Nicoara M (2014) Bioaccumulation of heavy metals in marine organisms from the Romanian sector of the Black Sea. N Biotechnol 32(3): 369-378.

11.Vinodhini R, Narayanan M (2008) Bioaccumulations of heavy metals in organs of fresh water fish Cyprinus carpio (Common carp). Int J Environ Sci Tech 5: 179-182.
This work is licensed under Creative Commons Attribution 4.0 License

Submission Link: Submit Article

DOI: $10.32474 / C D V S .2018 .02 .000131$

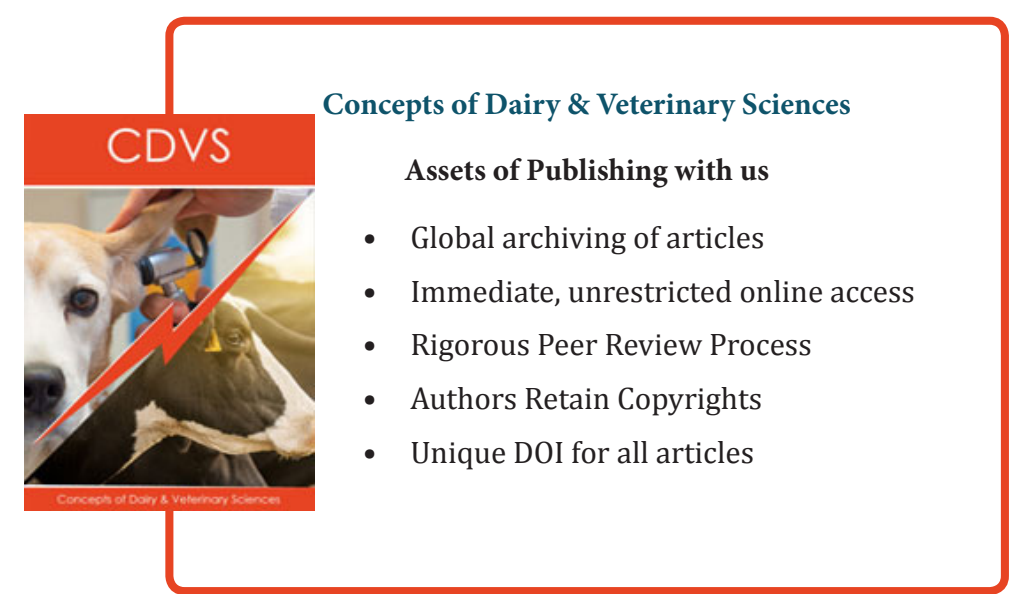

